\title{
Counterfactual thinking and decision making
}

\author{
NEAL ROESE \\ Northwestern University, Evanston, Illinois
}

\begin{abstract}
Recent research on counterfactual thinking is discussed in terms of its implications for decision making. Against a backdrop of the functional benefits of counterfactual thinking, two distinct types of bias, one liberal and one conservative, are discussed. Counterfactuals may cause decision makers to become liberally biased (i.e., capricious) in terms of tactics, but conservatively biased (i.e., rigid) in terms of long-term strategy. That is, counterfactuals may lead to short-term corrective changes that are needless and costly, but they may also lead to long-term overconfidence, blinding the decision maker to possible beneficial strategic adjustments. Recent research on counterfactual thinking, which is inherently multidisciplinary, is reviewed in light of a theoretical structure that posits two mechanisms by which counterfactual effects occur: contrast effects and causal inferences.
\end{abstract}

On May 1, 1960, an American U-2 spy plane was shot down over the Soviet Union, triggering an international crisis that torpedoed the emerging détente and the prospect of an arms reduction treaty. These events could be traced to a decision by President Eisenhower in mid April, 1960 , to send the U-2 spy plane into Soviet air space. The decision was not made lightly: Eisenhower weighed the advantages of insight into Soviet ballistic missile construction against the political fallout should the plane and its pilot be captured. After the fact, it is easy to wonder what might have happened had Eisenhower canceled rather than approved the May U-2 flight: The subsequent Paris summit might have produced an arms treaty, Eisenhower might have ended his term in triumph, and the costly arms buildup of the $1960 \mathrm{~s}$ might have been averted (Beschloss, 1986). Before a decision is made, a set of options constitute candidates for future reality. After the decision has been made and its consequences are known, those unchosen options can never become reality, but they may nevertheless haunt us, amuse us, or influence our perception of the decision process itself. The mental representations of events that might have occurred are termed counterfactuals, and an expanding body of research has linked their operation to a variety of judgmental consequences (Kahneman, 1995; Roese, 1997). This paper focuses on the link between counterfactuals and decision making, particularly from the vantage point of multiple decisions made serially and in parallel.

Throughout the twentieth century, counterfactuals have been discussed by philosophers (e.g., Lewis, 1973), historians (e.g., Ferguson, 1997), and political theorists

This project was supported by National Institute of Mental Health Grant MH55578, with thanks to Ashley Yirak for her clerical assistance. I thank Michael Morris, Jeff Sherman, and especially Doug Medin for valuable advice and discussion. Address correspondence to N. Roese, Department of Psychology, Northwestern University, Evanston, IL 60208-2710 (e-mail: roese@nwu.edu; URL: http://www.nwu.edu/ people/roese). (e.g., Tetlock \& Belkin, 1996). Empirical research on the production, storage, and retrieval of counterfactual inferences was conducted by cognitive psychologists beginning in the 1970s (e.g., Fillenbaum, 1974), but it was not until Kahneman and Tversky (1982) examined counterfactuals from the vantage point of biased decision making that psychological research on the topic mushroomed. Cued by this seminal work, social psychologists in the 1980s and 1990s assessed counterfactual thinking in terms of its implications for judgments such as blame and responsibility (e.g., Roese \& Olson, 1995). By the 1990 s, organizational behavior theorists were applying this research to real-world decision making (e.g., MeyersLevy \& Maheswaran, 1992) while cognitive psychologists continued to examine counterfactual thinking in light of deeper inferential processes, such as conditional, temporal, and causal reasoning (e.g., Byrne, 1997; Harris, German, \& Mills, 1996). Not surprisingly, then, findings regarding counterfactual thinking and decision making are scattered across disciplinary lines, appearing in numerous disparate journals. The goal of this article is to provide a theoretical overview of the role of counterfactuals in decision making, drawing on this diverse body of evidence while at the same time erecting a useful conceptual superstructure.

\section{Function Versus Bias}

Initial conceptions of the link between counterfactual thinking and decision making centered on the possible biasing effect of the former on the latter. More specifically, it was thought that judgments regarding relatively objective (e.g., monetary) outcomes could be deflected by the consideration of counterfactual outcomes. For example, Dale decides to buy stock in Intel, but then switches his portfolio over to Microsoft, only to learn that he would have been better off had he stayed with Intel. Realization that a better outcome might have occurred had some alternative decision been rendered (as opposed to the case in which no such information of alternative outcomes is 
available) results in a harsher evaluation of the decision and the decision maker (Kahneman \& Tversky, 1982). Put simply, a contrast effect has occurred, in that the negative factual outcome (and the path by which it emerged) seems even more negative when contrasted to a more favorable alternative outcome (Roese, 1997). Alternatives that are evaluatively better than actuality have been termed upward counterfactuals, whereas alternatives that are worse than actuality have been termed downward counterfactuals. Upward counterfactuals seem to be generated more frequently (Roese \& Olson, 1997) and have greater impact (Heath, Larrick, \& Wu, 1999) than downward counterfactuals do, and so the present discussion focuses mainly on the impact of upward counterfactuals. ${ }^{1}$ In general terms, then, the greater the salience of an upward alternative to a decision outcome, the more negatively the decision will be evaluated (Mellers, Schwartz, Ho, \& Ritov, 1997). Factors that elevate the relative salience of a counterfactual anchor may thus alter evaluations of past decisions and hence influence the unfolding of future decisions (see also Baron \& Hershey, 1988).

Such was the initial theoretical impetus provided by Kahneman and Tversky (1982). However, current research collectively suggests a wider framework within which the consequences of counterfactual thinking for decision making may be understood. Specifically, counterfactual thinking may also be functional (i.e., beneficial, useful) for ongoing problem solving and global behavior patterns, but can depart from functionality to produce bias in either of two distinct ways. The first way is a liberal bias, one that, as described above, leads to reappraisal of the decision itself and promotes an overactive tendency to make alterations within a series of related decisions, resulting in the implementation of perhaps costly changes. The second way is a conservative bias, one that centers on overconfidence that breeds a reluctance to make strategic alterations, particularly across multiple parallel domains of decision making. Although recent research has documented isolated components of these two effects, they have not until now been integrated into a unified framework. First to be considered is research on a functional basis of counterfactual thinking.

\section{Functionality}

A functional effect is generally denoted in terms of the fulfillment some specific purpose, as in, for example, functional analyses that link the structure of the cornea to the purpose of refracting light. Of course, functional need not mean "conscious" or "intentional"; evolution is one example of how function may be articulated in the absence of intelligent foresight. In the domain of achievement (e.g., career success, interpersonal relationship management), which constitutes the focus of most of our everyday counterfactual inferences, function is defined more specifically in terms of improvement in performance. That is, a functional process is one that serves the purpose of engendering improvement on some specific task. Counterfactual thinking has been understood by some as a functional cognitive process that occasionally produces judgmental bias (Markman, Gavanski, Sherman, \& McMullen, 1993), but the variables that predict functionality versus bias have not been well delineated.

Demonstrations of counterfactual functionality have focused on achievement, with tasks ranging from games of chance to verbal puzzles to college exam performance. A key postulate is that causal information is an inferential offshoot of counterfactual thinking. A counterfactual conditional (e.g., "If Dana had studied, she would have passed the exam") contains information that links an antecedent to an outcome. If a counterfactual antecedent (e.g., studying more) seems to account for the discrepancy between a factual outcome (e.g., failing) and a counterfactual outcome (e.g., passing), this antecedent is then perceived to be causally important. Such causal information, to the extent that it is accurate, may then be deployed to enhance future performance: Dana might plan to study in the future.

Three lines of research support the functional role of counterfactuals. First, manipulations of counterfactual thoughts do indeed produce specific effects on causal inferences (Harris et al., 1996; Wells \& Gavanski, 1989). That is, the antecedent within a counterfactual conditional is generally seen to be more causally potent to the extent that the counterfactual itself becomes salient. ${ }^{2}$ Second, manipulations of counterfactual thinking influence performance on achievement tasks. For example, relative to a no-counterfactual control condition, participants instructed to generate counterfactuals regarding a computer-administered anagram task subsequently perform better on a second anagram task (Roese, 1994). Similarly, student pilots are more successful in computersimulated landing attempts after generating versus not generating counterfactuals centering on their past performance (Morris \& Moore, 1998). Third, counterfactual thinking is uniquely activated by situations that demand corrective action, such as those involving failure or threat. Upward counterfactuals are more frequently activated by situations that are negative (Sanna \& Turley, 1996), by negative emotions (Roese \& Olson, 1997), and by serially problematic situations (Markman et al., 1993). In general, cognitions aimed at problem solving are mobilized automatically in response to negative affect or perceived threat (Taylor, 1991), and counterfactual thinking is a cogent example of this general pattern.

A decision followed by a negative consequence normally evokes counterfactual musings centering on alternative but foregone decision options. Under many circumstances, counterfactual thinking may underscore or clarify causal inferences, thereby paving the way for subsequent changes that can result in improvement. It is against this functional backdrop, which describes the typical effects of counterfactual thinking, that delineation of counterfactual biases are best understood (Arkes, 1991). Moreover, nearly all demonstrations of counterfactual effects have been rooted to either a contrasi effect mechanism or a causal inference mechanism (Roese, 1997). Recognition of the contrast effect mechanism 
helps to illuminate the liberal bias, whereas recognition of the causal inference mechanism helps to illuminate the conservative bias.

\section{Liberal Bias}

A liberal bias is an increase in alterations over multiple decision episodes. This assumes an optimal degree of flexibility in the course of serial decision making, such that monetary (or other) outcomes may be maximized. More important, it assumes that decision alterations are optimal because they are effective (i.e., they produce the intended consequence). If a counterfactual incorrectly suggests that a specific decision option would have brought about a specific outcome, then applying this counterfactual to a subsequent decision would be ineffective. The subsequent decision might make matters worse or it might have no effect; in either case, the cost of implementation will be wasted.

As one illustration, after realizing the negative consequences of his decision to approve the May U-2 flight, each of Eisenhower's subsequent decisions regarding $U$ 2 flights were refusals rather than approvals. In this case, the counterfactual likely rendered an accurate causal inference, and in such cases it is to the decision maker's advantage to decide differently next time. On the other hand, when the counterfactual suggests a specious causal inference, subsequent decision change is harmful. A novice investor, realizing that better profits might have been made elsewhere, might sell off stock, but this overactive tendency to change course will likely be suboptimal (i.e., financial experts argue that novice investors switch their investments too often). The overactive tendency to alter serial decisions in this case produces no gain in profit while wasting money on brokerage fees.

Process underlying liberal bias. The specific pathway of effects for the liberal bias is as follows. First, a counterfactual is constructed in response to an undesirable decision consequence. Second, by way of a contrast effect, the counterfactual makes an obtained decision and its outcome appear even less satisfactory. And third, relatively greater dissatisfaction prompts relatively greater decision change, such that the next decision in a serial chain is more likely to be reassessed and a different option selected. Evidence pertaining to the second and third components is considered first, followed by a description of two determining variables that influence whether a liberal bias rather than a functional effect appears.

General evidence. Evidence that counterfactual thinking influences satisfaction, particularly when it takes the form of an upward comparison, is wide ranging. For example, Markman et al. (1993) showed that gambling decisions that resulted in a loss created greater disappointment as a function of the salience of missed opportunities to win. Medvec, Madey, and Gilovich (1995) showed that salient upward counterfactual comparisons among Olympic medalists (i.e., when a silver medallist imagines what she might have done to have won the gold) reduced their satisfaction with their performance, relative to control participants. By manipulating the value of the outcome of alternative gambling decisions, Mellers et al. (1997) showed a roughly linear relation, such that upward alternatives of increasing value bring about increasing degrees of regret. In all these demonstrations, the common element is an affective contrast effect in which an upward comparison anchor makes a factual outcome appear relatively less desirable (Roese, 1997). Such a pattern is one example of the more general class of "framing effects" that have been studied under the rubric of biased decision making (Kahneman \& Tversky, 1984).

Second, dissatisfaction prompts behavior change. At the most basic level is the pattern that negative affect activates avoidance behavior and problem-solving cognitions, at least relative to neutral and positive affect (see Taylor, 1991, for a multidisciplinary review). A more specific theoretical depiction of this effect is relative deprivation theory (Crosby, 1976), which seeks to specify the conditions under which social inequity unleashes protest behavior. Although the full range of this theory is beyond the scope of the present discussion, the most relevant point is that dissatisfaction alters subsequent decisions and produces changes in behavior, such as when one seeks new employment, obtains extra training, alters managerial style, and so forth (Folger, Rosenfield, Rheaume, \& Martin, 1983; see Olson \& Hafer, 1996, for a recent review). Within the counterfactual literature, Roese (1994) has shown directly that a manipulation of counterfactual thinking influenced tactical change. Participants completed two discrete blocks of anagrams, separated in time by the counterfactual induction. Participants induced to generate upward counterfactuals (relative to downward counterfactuals and control participants) were more likely to alter their tactics on the task; for example, they reduced the number of solution "clues" that they "bought" in the subsequent block of anagrams. These various strands of evidence suggest that counterfactually induced negative affect prompts tactical change within serial decision making.

An experimental demonstration. One recently reported program of research has assembled these components together to demonstrate a counterfactual-induced liberal bias in serial decision making (Pfister, van der Pligt, \& van Dijk, 1999). Before beginning a general-knowledge test, participants selected either a cautious or a risky version of the test, defined in terms of payoffs contingent on their performance. In one condition, participants received failure feedback regarding their own outcome, whereas in the counterfactual feedback condition, participants received failure feedback plus information indicating a clearly better payoff had they chosen the other option. Affect (e.g., regret and disappointment) and decision change (i.e., whether they would alter their selection if they were taking the test again) were measured both immediately and 1 week after receipt of feedback. Generally speaking, findings were similar at both points in time.

The counterfactual feedback condition produced heightened regret and also heightened decision change relative 
to the control condition. Liberal bias took the form of relative insensitivity to utility. Specifically, regret (an emotion uniquely associated with counterfactual thinking; see Zeelenberg et al., 1998) predicted decision change that occurred regardless of whether the shift was toward a cautious versus a risky option. Disappointment (which is not uniquely associated with self-focused counterfactual thinking) predicted shifts only toward the more cautious choice. These findings indicate that counterfactual thinking per se can push people toward decision change with reduced attention to other useful sources of information (see also Zeelenberg \& Beattie, 1997, Experiment 3).

Determinants of liberal bias. An essential ingredient of the liberal bias is that under some circumstances, counterfactuals suggest causal inferences that are specious, and hence decision change made in light of them results in no subsequent improvement. What we are dealing with, then, is the functional process, described in the previous section, deflected by incidental informational cues. The determining variables that influence whether counterfactual thinking produces a liberal bias versus a functional effect are essentially incidental cues that magnify the affective contrast effect but do not contain any useful insight for causal inference (Sherman \& McConnell, 1996). Many such variables have been examined in research over the last 15 years, but two particularly prominent ones are reviewed here: proximity and serial position.

Kahneman and Tversky (1982) first discussed the variable of proximity (or closeness) of a counterfactual to having achieved factual status. People often believe that some outcomes "nearly" happened whereas others never came close to actuality, particularly when the former show a propensity or acceleration toward, but are thwarted just before becoming, an actual occurrence (Kahneman \& Varey, 1990). Incidental information that suggests proximity can activate counterfactual thoughts. In one experiment, exposure to information that a stranded traveler who died of exposure just a few hundred yards from safety (as opposed to many miles from safety) produced larger counterfactual effects (Miller \& McFarland, 1986, Experiment 2). Proximity can be defined temporally as well as spatially. In the earliest and most cited demonstration, Kahneman and Tversky (1982) obtained greater affective contrast effects in response to a target individual who missed a plane by $5 \mathrm{~min}$ than in responses to another traveler who missed a flight by an hour. Most important in terms of the evocation of the liberal bias, the experimental manipulation of counterfactual proximity produces affective contrast, but at the same time has no informational value for causal insight (Roese \& Olson, 1996, Experiment 3 ). That is, proximity activates counterfactual thinking without illuminating accurate causal inferences. Metaphorically speaking, the vehicle has embarked without a driver.

Serial position as a determining variable is similar to proximity in that it also makes counterfactuals salient without necessarily imbuing them with accurate causal information. In a chain of events prior to an outcome, perceivers tend to focus their counterfactuals on antecedents that occur later rather than sooner. This effect is particularly apparent when the events in a chain are not clearly connected causally, such as when a sequence of independent coin tosses collectively determine a winning outcome (e.g., Miller \& Gunasegaram, 1990). In two experiments, Sherman and McConnell (1996) presented to participants descriptions of basketball outcomes in which serial events were manipulated. A direct measure of counterfactual thinking supported the conclusion that counterfactuals are more likely to center on the alteration of later rather than earlier events (e.g., the final free throw in a close game rather than points scored earlier in the game). Other research indicates that this serial position effect can be pinpointed more exactly by specifying, in addition to counterfactual reasoning, probabilistic inferences (Spellman, 1997) and mental models (Byrne, 1997). Nevertheless, these later elaborations are compatible with the more basic conclusion that serial position can influence the activation of counterfactuals without pushing them toward more accurate causal insights.

Summary. These lines of research collectively suggest that decision makers who recognize upward counterfactual possibilities may sometimes fall prey to a liberal bias, which increases decision change within serial decision episodes. If the decision change is predicated on faulty counterfactual-derived causal inference, the decision change will be useless. Variables that activate liberally biased counterfactual thinking, such as proximity and serial position, do so because they constitute informational cues that magnify counterfactual consequences based on an affective contrast effect without at the same time producing accurate causal inferences. The ideas summarized in this section comprise the status quo in the judgment and decision-making literature. The next section, by contrast, describes newer insights suggesting that counterfactuals may evoke bias in a manner quite distinct from that detailed thus far.

\section{Conservative Bias}

A conservative bias centers on a reduction in alterations made over multiple decisions. If a liberal bias is an increase in alterations over multiple decisions, how can counterfactual thinking simultaneously produce both? Multiple decisions can be viewed as a serial set of relatively domain-similar decisions recurring over time (as in the case of Eisenhower's repeated decisions regarding U-2 flights into the Soviet Union between 1956 and 1960), but they can also be viewed as a set of domain-diverse decisions made in parallel by the same decision maker (as in the case of Eisenhower's many other foreign policy decisions). More precisely, liberal bias occurs at the tactical level, which embraces serial decision making occurring over a relatively short period of time. Conservative bias, on the other hand, occurs at the strategic level, 
which embraces not only a longer time frame but also many other decision streams occurring in parallel with the focal decision.

The theoretical basis of a conservative bias ties together research on overconfidence (e.g., Fischhoff, Slovic, \& Lichtenstein, 1977; Vallone, Griffin, Lin, \& Ross, 1990) with that on counterfactual thinking. A counterfactual has (in addition to the effects already described in previous sections) the additional effect of evoking a self-descriptive inference about one's own decision-making prowess, such as, "I am a good decision maker and I know exactly what I am doing." Specifically, the counterfactual illuminates a causal inference that details means of improvement, and because this counterfactual centers on one's own decisions, it underscores the presumed effectiveness of one's own actions. As a result, overconfidence in the form of self-perceptions of mastery, competence, and self-efficacy are heightened (Bandura, 1997; Nasco \& Marsh, 1999). Such overconfidence may then result in disengagement-that is, in a "hands off" policy in which attention to detail is curtailed. With this, alteration to parallel decisions becomes less likely, as creative avenues that might address problems or bring about long-term improvement are ignored.

Such conservatism born of overconfidence has consequences not so much for the evoking decision's domain, but rather for a broader set of parallel decisions. For example, to the extent that Eisenhower became overconfident through musings of how the U-2 crisis might have been avoided, he might then focus less attention and expend less effort on other decisions, such as whether to continue covert operations in Cuba, disengage from Laos, or explore new treaty options with China.

Process underlying conservative bias. The specific pathway of effects for the conservative bias is as follows. First, counterfactual thinking influences causal inference (Wells \& Gavanski, 1989). Second, causal insight may produce overconfidence (Anderson, Lepper, \& Ross, 1980), defined in terms of global self-perceptions of confidence. That is, if counterfactual thinking leads one to focus on one's own decisions, then one offshoot inference is that one is personally potent in terms of power to bring about changed outcomes. In other words, perceptions of personal control and self-efficacy are heightened by self-focused counterfactual thinking (Nasco \& Marsh, 1999). Third, overconfidence reduces attention to procedural detail, which in turn reduces the likelihood of making decision alterations. Evidence for the first link (counterfactual effects on causal inference) have already been discussed. Recent research on the link between counterfactual thinking and overconfidence comes from a particular judgmental effect termed the hindsight bias.

Hindsight bias. The hindsight bias is a tendency to see past events as predictable and sensible relative to predictions of events made before their occurrence (see Hawkins \& Hastie, 1990, for a review). Typically, the hindsight bias has been studied by decision theorists who view it as a particularly illuminating case of overconfi- dence. The more clearly an event can be explained in retrospect, the more inevitable it may come to be seen (Fischhoff, 1982). Thus, experimental manipulations of the quality or clarity of causal explanation influences hindsight confidence (Wasserman, Lempert, \& Hastie, 1991). Any counterfactual conditional that yields causal information should also, by this same logic, produce overconfidence (cf. Koriat, Lichtenstein, \& Fischhoff, 1980). For example, a sports fan might react to his or her team's loss with the counterfactual judgment that the team would have won were it not for an injury suffered in the third quarter. Without that injury, a victory would have been assured. In this way, the conditional counterfactual points to some causal feature that accounts for the outcome, yielding a satisfying feeling of explanation, comprehension, and post hoc certainty.

One recent set of experiments supports this reasoning (Roese \& Olson, 1996). Participants read a scenario describing a student preparing for but then performing poorly on an important exam. For half of the participants, the poor performance was easily "undone" mentally through the imagined alteration of a salient antecedent action performed by the student. For the other participants, altering this antecedent action would not have changed the outcome. The hindsight bias was greater for the participants who could mentally undo the outcome. Parallel effects were obtained on a measure of causal inferences: The target antecedent action was seen as more causally important when altering this antecedent would have undone the outcome (Roese \& Olson, 1996, Experiment 1). In the third experiment reported in that article, causal inferences were shown to mediate the impact of counterfactual thinking on the hindsight bias. In other words, counterfactuals inflate confidence in one's decisionmaking prowess only to the extent that the counterfactual proffers a causal explanation that satisfyingly accounts for the outcome at hand (see also Roese \& Maniar, 1997).

A related form of conservative bias is a tendency to "explain away" negative outcomes by attributing them to external rather than internal factors (Miller \& Ross, 1975). In other words, rather than drawing conclusions about procedural flaws, decision makers often attribute failure to causes that are external and unstable-factors that are extrinsic to the decision maker. For example, corporate managers often explain poor performance to stockholders by pointing to capricious economic events that operate far outside the control of the managers themselves (Staw, McKechnie, \& Puffer, 1983). Recently, Tetlock (1998) expanded upon this general finding by showing unique patterns of counterfactual thinking deployed in the defense of political belief systems. Historical and political experts, when confronted with errors of prediction (e.g., failure to predict the collapse of the Soviet Union) were found to embrace counterfactuals that in some way bolstered their particular ideological vantage point (e.g., the 1991 coup attempt almost led to a continuation of the Soviet Union). In other words, they claimed that they were "almost right" and constructed counter- 
factuals to support this belief. In this way, experts were able to persist in their views despite the appearance of evidence contradicting their views.

Summary. In the discussion of liberal bias above, an important issue was when, or under what circumstances, a liberal bias rather than a functional effect would obtain. For a conservative bias, the issue is not when but where. That is, a conservative bias has been conceptualized not as an alternative to a functional effect, but rather as a higher order consequence that is superimposed over a functional effect. To summarize, a conservative bias, then, is one that centers on resistance to procedural modification, but it occurs at a strategic level and may center on multiple decisions rendered in parallel to the focal decision. Counterfactual thinking, by underscoring causal perceptions that contribute to confidence in one's understanding of the past, may give rise to overconfident selfperceptions of mastery, which then yield strategic rigidity. The evidence is fairly clear on the link between counterfactuals, causal inference, and overconfidence. However, experimental evidence that links these variables to procedural rigidity remains to be demonstrated. This framework therefore suggests some clear directions for future research.

\section{Tactics Versus Strategy in Decision-Making}

To reiterate the ideas presented so far, we have seen that counterfactuals might evoke alterations in decision making that are liberal or conservative. More generally, counterfactual thinking produces effects that are undergirded by contrast effects and causal inferences. Finally, decision making can be viewed at a tactical level or at a strategic level. In this section, the connections between these concepts are clarified further.

To borrow from military theory, tactics and strategy are best defined not absolutely but rather in relation to each other. The strategic level centers on winning the waron the highest order, longest term goal. The tactical level is a looser subdivision of the war's duration into at least two but as many as several dozen overlapping episodes, each of which has a clear beginning and end. An additional feature that helps to identify the scope of effects is whether multiple decisions are viewed serially or in parallel. Tactical may refer to a set of successive decisions rendered within a particular domain of interest, whereas strategic may refer to multiple decisions, made in parallel, that span numerous domains.

I have argued that liberal bias centers on a lower, briefer, tactical level of decision making, whereas conservative biases center on a higher, wider, strategic level of decision making. Thus, liberal bias operates at the level of discrete decisions and outcomes, and is compatible with the argument that counterfactuals are constructed online uniquely in light of each evoking outcome (Kahneman \& Miller, 1986). Conservative bias, on the other hand, is tied to general perceptions of one's overarching abilities, and thus can have broad effects across numerous decision domains. In a previous review article (Roese,
1997), I argued that most of the wide-ranging effects of counterfactual thinking could be reduced to one of two mechanisms: contrast effects and causal inferences. The liberal bias is rooted to the contrast effect mechanism, in that incidental information may invite counterfactual comparisons that then produce bias through an affective contrast effect. The conservative bias, on the other hand, is rooted to the causal inference mechanism, in that higher order self-perceptions that are overconfident stem from counterfactually induced self-focused causal inferences. Table 1 summarizes these ideas.

This suggests that the liberal versus conservative biases do not constitute opposing poles on a dimension of "decision change." Rather, it suggests that functionality and liberal bias are closely paired, in that they both operate at a tactical level and involve alterations to successive decisions within a domain-specific serial chain. Conservative bias, on the other hand, stands apart, with effects operating at the higher level of self-perception. If conservative bias is rooted to inflation of self-perceptions of efficacy and mastery, such perceptions can influence a wide variety of decisions situated in entirely different domains. Thus, we are faced with a disjunction between the nature of functionality and liberal bias, on the one hand, and conservative bias, on the other, in terms of both temporal scale and conceptual scale. This theoretical demarcation of effects may suggest insights into efforts to reduce biased decision making.

\section{Implications for Reduction of Bias}

Few theorists have considered the prospect of designing interventions to reduce counterfactual thinking with the goal of curtailing decision bias. In part this has been due to an assumption that counterfactual thinking is an integral component of human cognition (e.g., Hofstadter, 1979; see pp. 633-644). Although this may be true in a general sense, it remains possible that counterfactual thinking may be modified and controlled so as to facilitate decision-making performance in more specific contexts. One way to design interventions is to take into account the respective processes and variables involved in liberal versus conservative bias. This section is a brief overview of some previously published suggestions for reduction of bias in decision making, reinterpreted in light of the theoretical framework of this paper.

An intervention designed to combat a liberal bias could focus on counteracting the effect of incidental information, such as that involving proximity and serial position, that makes certain counterfactual comparisons relatively more salient. If specific decisions may be shifted arbitrarily by any one anchor (Kahneman \& Tversky, 1984),

\section{Table 1}

Liberal Versus Conservative Bias

\begin{tabular}{lll}
\hline & Liberal Bias & Conservative Bias \\
\hline Mechanism & Contrast effect & Causal inference \\
Temporal frame & Tactical & Strategic \\
Decision domain & Serial & Parallel \\
\hline
\end{tabular}


the imposition of a more representative sample of anchors might reduce the liberal bias (Boles \& Messick, 1995; Kahneman, 1992). A counterfactual representation can have an anchoring effect, such that the salience of the anchor dictates the subsequent directional deflection of judgments. But if several anchors were equivalently salient, and if these pulled the judgment in opposite directions, the net judgmental shift might be reduced or eliminated (cf. Hirt \& Markman, 1995). To use a different metaphor, a combination of opposing forces produces a state of equilibrium with zero net movement. For example, a coach might evaluate his team's loss in a close game in light of the victory that he narrowly missed by a handful of points. This single upward comparison anchor makes the game outcome less satisfactory, by contrast. But by considering a variety of alternative point spreads, including those that would be worse than what actually happened, the impact of the previously mentioned contrast effect would be mitigated.

Another strategy to reduce contrast effects is to shunt the pathway away from a contrast effect and toward an assimilation effect. McMullen (1997) produced counterfactual-induced assimilation effects in two laboratory experiments. This was achieved by directing participants' attention toward the experiential aspects of an alternative self-relevant outcome, as in daydreaming about being rich enough to own a yacht. In this case, a direct comparison between factual and counterfactual is suspended while features of the counterfactual are examined in relative isolation. This isolated examination produced affective assimilation rather than contrast. Whether this technique may be applied profitably to real life decisionmaking situations remains for future research to answer.

An intervention that would influence both tactical liberal bias and also strategic conservative bias would target the accuracy of causal inferences derived from counterfactual thinking. Unfortunately, the best prescription for an accurate understanding of causality is to run an experiment, nearly always an impossibility in everyday life. Moreover, many research projects have documented the inaccuracy of lay causal reasoning (Gilbert, 1998; Nisbett \& Ross, 1980), suggesting a pervasive pattern that is relatively immune to quick fixes. However, there might be some general tools with which the inaccuracy of causal judgments may at least be lessened. For example, because human beings tend toward parsimony in explanation, seizing on the first casual conclusion for a given event that comes their way, they tend to consider only a tiny subset of all possible conclusions (see, e.g., Shaklee \& Fischhoff, 1982). Once a single causal factor is initially recognized, it may be elevated to the status of "sole cause" even though further consideration might yield a different conclusion. Interventions designed to force the decision maker to ponder other combinations of specific decisions with specific alternative outcomes might alter causal conclusions. Thus, consideration of the role of al- ternative factors that were similarly sufficient to alter a given outcome would weaken the perception that the initially identified cause was important (Morris \& Larrick, 1995). Or, consideration of the frequency with which the factual outcome occurred in the absence of the identified causal factor would also weaken causal inferences centering on that factor, thus reducing overconfidence.

These suggestions for interventions designed to reduce either liberal or conservative biases are of course highly speculative. Yet the theoretical framework suggested here may establish a road map for future researchers who wish to address some of these possibilities.

\section{Conclusion}

The link between counterfactual thinking and decision making is close. Any assessment of the quality of a given decision will be dictated by mental simulations of what might have happened had an alternative decision been made. The multidisciplinary literature on counterfactual thinking is therefore of great relevance to contemporary theorists of decision making. My goal in the present review has been to underscore some recent findings in the area of counterfactual thinking and how they clarify the processes by which multiple decision making might be biased. Counterfactuals can evoke a liberal bias, which amounts to more negative appraisals of the initial decision and a tendency toward costly changes in ongoing procedure. But they can also evoke a conservative bias, in which perceptions of personal causal efficacy lead to overconfidence. The liberal bias is rooted in a contrast effect mechanism and operates at the tactical level, whereas the conservative bias is rooted in a causal inference mechanism and operates at the strategic level. This theoretical framework, within which the variegated judgmental consequences of counterfactual thinking may be understood, may donate greater clarity to future attempts to both understand and reduce bias in decision making.

\section{REFERENCES}

ANDERson, C. A., LePper, M. R., \& Ross, L. (1980). Perseverance of social theories: The role of explanation in the persistence of discred ited information. Journal of Personality \& Social Psychology, 39. 1037-1047.

ARKES, H. R. (1991). Costs and benefits of judgment errors: Implications for debiasing. Psychological Bulletin, 110, 486-498.

Bandura, A. (1997). Self-efficacy: The exercise of control. New York: W. H. Freeman.

Baron, J., \& Hershey, J. C. (1988). Outcome bias in decision evaluation. Journal of Personality \& Social Psychology, 54, 569-579.

Beschloss, M. R. (1986). May-Day: Eisenhower, Khrushchev, and the U-2 affair. New York: Harper \& Row.

Boles, T. L., \& Messick, D. M. (1995). A reverse outcome bias: The influence of multiple reference points on the evaluation of outcomes and decisions. Organizational Behavior \& Human Decision Processes, 61, 262-275.

ByRne, R. M. J. (1997). Cognitive processes in counterfactual thinking about what might have been. In D. L. Medin (Ed.), The psychology of learning and motivation: Advances in research and theory (Vol. 37, pp. 105-154). San Diego: Academic Press. 
Crosby, F. (1976). A model of egoistical relative deprivation. Psychological Review, 83, 85-113.

FERGUSON, N. (ED.) (1997). Virtual history: Alternatives and counterfactuals. London: Picador.

Fillenbaum, S. (1974). Information amplified: Memory for counterfactual conditionals. Journal of Experimental Psychology, 102, 44-49

Fischroff, B. (1982). For those condemned to study the past: Heuristics and biases in hindsight. In D. Kahneman, P. Slovic, \& A. Tversky, (Eds.), Judgment under uncertainty: Heuristics and biases (pp. 335-351). New York: Cambridge University Press.

Fischhoff, B., Slovic, P., \& Lichtenstein, S. (1977). Knowing with certainty: The appropriateness of extreme confidence. Journal of Experimental Psychology: Human Perception \& Performance, 3, 552-564.

Folger, R., Rosenfield, D., Rheaume, K., \& Martin, C. (1983). Relative deprivation and referent cognitions. Journal of Experimental Social Psychology, 19, 172-184.

GilberT, D. T. (1998). Ordinary personology. In D. T. Gilbert, S. T. Fiske, \& G. Lindzey (Eds.), Handbook of social psychology (4th ed.; Vol. 2, pp. 89-150). New York: McGraw-Hill.

Harris, P. L., German, T., \& Mills, P. (1996). Children's use of counterfactual thinking in causal reasoning. Cognition, 61, 233-259.

Hawkins, S. A., \& HastiE, R. (1990). Hindsight: Biased judgment of past events after the outcomes are known. Psychological Bulletin, 107, 31 1-327.

Heath, C.. Larrick, R. P., \& WU. G. (1999). Goals as reference points. Cognitive Psvchology, 38, 79-109.

HIRT, E. R., \& MARKMAN, K, D. (1995). Multiple explanation: A consider-an-alternative strategy for debiasing judgments. Joumal of Personality \& Social Psychology, 69. 1069-1086.

Hofstadter, D. R. (1979). Gödel, Escher, Bach: An eternal golden braid. New York: Vintage Books.

KahNEMAN, D. (1992). Reference points, anchors, norms, and mixed feelings. Organizational Behavior \& Human Decision Processes, 51, 296-312.

KahNemAN, D. (1995). Varieties of counterfactual thinking. In N. J. Roese \& J. M. Olson (Eds.), What might have been: The social psychologv of counterfactual thinking (pp. 375-396). Mahwah, NJ: Erlbaum.

Kahneman, D., \& Miller, D. T. (1986). Norm theory: Comparing reality to its alternatives. Psychological Review, 93, 136-153.

KahNeman, D., \& TVERSKY, A. (1982). The simulation heuristic. In D. Kahneman, P. Slovic, \& A. Tversky (Eds.), Judgment under uncertainty: Heuristics and biases (pp. 201-208). New York: Cambridge University Press.

Kahneman, D.. \& Tversky, A. (1984). Choices, values, and frames. American Psychologist, 39, 341-350

Kahneman, D., \& Varey, C. A. (1990). Propensities and counterfactuals: The loser that almost won. Journal of Personality \& Social Psycholog $v, 59,1101-1110$.

Koriat, A., Lichtenstein, S., \& Fischhoff, B. (1980). Reasons for confidence. Journal of Experimental Psychology: Human Learning \& Memorv, 6, 107-118

LEwIs, D. (1973). Counterfactuals. Cambridge, MA: Harvard University Press.

Markman, K. D., Gavanski, I., Sherman. S. J., \& McMullen, M. N (1993). The mental simulation of better and worse possible worlds Journal of Experimental Social Psychology, 29, 87-109.

MCMuLLEN, M. N. (1997). Affective contrast and assimilation in counterfactual thinking. Journal of Experimental Social Psychology, 33 77-100.

Medvec, V. H., Madey, S. F., \& Gilovich, T. (1995). When less is more: Counterfactual thinking and satisfaction among Olympic athletes. Journal of Personality \& Social Psychologv, 69, 603-610.

Mellers, B. A., Schwartz, A., Ho, K., \& Ritov, I. (1997). Decision affect theory: Emotional reactions to the outcomes of risky options Psychological Science, 8, 423-429.

Meyers-LeVy, J., \& Maheswaran, D. (1992). When timing matters: The influence of temporal distance on consumers' affective and persuasive responses. Journal of Consumer Resear'h, 19, 424-433.

Miller, D. T., \& Gunasegaram, S. (1990). Temporal order and the perceived mutability of events: Implications for blame assignment. Journal of Personality \& Social Psychology, 59, $1111-1118$.
Miller, D. T., \& MCFarland, C. (1986). Counterfactual thinking and victim compensation: A test of norm theory. Personality \& Social Psychology Bulletin, 12, 513-519.

Muller, D. T., \& Ross, M. (1975). Self-serving biases in the attribution of causalilty: Fact or fiction? Psychological Bulletin, 82, 213-225.

Morris, M. W., \& LARrick, R. P. (1995). When one cause casts doubt on another: A normative analysis of discounting in causal attribution. Psychological Review, 102, 331-355.

MorrIS, M. W., \& Moore, P. C. (1998). Learning from a brush with danger: Evidence that pilot learning from dangerous incidents is enabled by counterfactual thinking and hindered by organizational accountability (Working Paper No. 1492). Stanford University, Graduate School of Business.

Nasco, S. A., \& Marsh, K. L. (1999). Gaining control through counterfactual thinking. Personality \& Social Psychology Bulletin, 25, 556-568.

NiSBetT, R. E., \& Ross, L. (1980). Human inference: Strategies and shortcomings of social judgment. Englewood Cliffs, NJ: Prentice-Hall.

Olson, J. M., \& HAFER, C. L. (1996). Affect, motivation, and cognition in relative deprivation research. In R. M. Sorrentino \& E. T. Higgins (Eds.), Handbook of motivation and cognition: The interpersonal context (Vol. 3, pp. 85-117). New York: Guilford.

Pfister, H. R., van der Pligt, J., \& van Dijk, W. (1999). Temporal aspects of regret and disappointment and their impact on choice behavior. Manuscript submitted for publication.

ROESE, N. J. (1994). The functional basis of counterfactual thinking. Journal of Personality \& Social Psychology, 66, 805-818.

RoEsE, N. J. (1997). Counterfactual thinking. Psychological Bulletin, 121, 133-148.

Roese, N. J., \& Maniar, S. D. (1997). Perceptions of purple: Counterfactual and hindsight judgments at Northwestern Wildcats football games. Personality \& Social Psychology Bulletin, 23, 1245-1253.

RoEsE, N. J., \& OLson, J. M. (EDs.) (1995). What might have been: The social psychology of counterfactual thinking. Mahwah, $\mathrm{NJ}$ : Erlbaum.

RoESE, N. J., \& Olson, J. M. (1996). Counterfactuals, causal attributions, and the hindsight bias: A conceptual integration. Journal of Experimental Social Psychology, 32, 197-227.

Roese, N. J., \& Olson, J. M. (1997). Counterfactual thinking: The intersection of affect and function. In M. P. Zanna (Ed.), Advances in experimental social psychology (Vol. 29, pp. 1-59). San Diego: Academic Press.

SanNa. L. J., \& Turley, K. J. (1996). Antecedents to spontaneous counterfactual thinking: Effects of expectancy violation and outcome valence. Personality \& Social Psychology Bulletin, 22, 906-919.

Sanna, L. J., Turley-Ames, K. J., \& Meier, S. (1999). Mood, selfesteem, and simulated alternatives: Thought-provoking affective influences on counterfactual direction. Journal of Personality \& Social Psychology, 76, 543-558.

SHAKLEE, H., \& FischHOFF, B. (1982). Strategies of information search in causal analysis. Memory \& Cognition, 10, 520-530.

Sherman, S. J., \& MCConnell, A. R. (1996). The role of counterfactual thinking in reasoning. Applied Cognitive Psychology, 10, S113-S124.

SPELLMAN, B. A. (1997). Crediting causality. Journal of Experimental Psychology: General, 126, 323-348.

Spellman, B. A., \& Mandel, D. R. (1999). When possibility inforns reality: Counterfactual thinking as a cue to causality. Current Directions in Psychological Science, 8, 120-123.

Staw, B. M., McKechnie, P. I., \& Puffer, S. M. (1983). The justification of organizational performance. Administrative Science Quarterly, 28, 582-600.

TAYLOR, S. E. (1991). Asymmetrical effects of positive and negative events: The mobilization-minimization hypothesis. Psychological Bulletin, 110, 67-85.

TetLOCK, P. E. (1998). Close-call counterfactuals and belief system defense: I was not almost wrong but I was almost right. Journal of Personality \& Social Psychology, 75, 639-652.

Tetlock, P. E., \& Belkin, A. (EDs.) (1996). Counterfactual thought experiments in world politics. Princeton, $\mathrm{NJ}$ : Princeton University Press.

Vallone, R. P., Griffin, D. W.. Lin, S., \& Ross, L. (1990). Overconfident prediction of future actions and outcomes by self and others. Journal of Personality \& Social Psychology, 58, 582-592. 
Wasserman, D., Lempert, R. O., \& Hastie, R. (1991). Hindsight and causality. Personality \& Social Psychology Bulletin, 17, 30-35.

Wells, G. L., \& GavansKI, I. (1989). Mental simulation of causality. Journal of Personality \& Social Psychology, 56, 161-169.

ZeElenberG, M., \& BeatTIE, J. (1997). Consequences of regret aversion 2: Additional evidence for effects of feedback on decision making. Organizational Behavior \& Human Decision Processes, 72, 63-78.

Zeelenberg, M., van DiJk, W. W., van der Pligt, J., Manstead, A. S. R., van Empelen, P., \& Reinderman, D. (1998). Emotional reactions to outcomes of decisions: The role of counterfactual thinking in the experience of regret and disappointment. Organizational Behavior \& Human Decision Processes, 75, 117-141.

\section{NOTES}

1. Evidence that upward counterfactuals are the default response comes from several sources. Coded open-ended counterfactual re- sponses to thought-listing questions are nearly always upward (Roese \& Olson, 1997). Some individuals may generate downward counterfactuals deliberately as a mood-enhancement strategy, but this tendency is reduced when attention is redirected elsewhere (Sanna, Turley-Ames, \& Meier, 1999). Explicit realization of a near miss or close call may evoke downward counterfactuals (McMullen, 1997), but this seems to occur relatively infrequently. Nevertheless, with the generation of downward counterfactuals, some of the effects described here might be reversed. 2. The conclusion that counterfactuals are sufficient to influence the magnitude of specific causal inferences should not be confused with the more general but empirically unsupported claim that counterfactuals are necessary for causal inferences to be drawn (cf. Roese, 1997; Spellman \& Mandel, 1999).

(Manuscript received July 16, 1998 ; revision accepted for publication July 27,1999 .) 